\title{
HCCS1 overexpression induces apoptosis via cathepsin D and intracellular calcium, and HCCS1 disruption in mice causes placental abnormality
}

\author{
Y Gan ${ }^{1}, \mathrm{X} \mathrm{Zhao}^{2}, \mathrm{~J} \mathrm{Hu}^{1}, \mathrm{ZG}$ Wang $^{\star, 2}$ and XT Zhao ${ }^{\star, 1}$
}

Hepatocellular carcinoma suppressor 1 (HCCS1) was discovered as a novel tumor suppressor gene. We recently observed that adenovirus-mediated gene transfer of HCCS1 leads to cytotoxicity to human hepatocarcinoma cells. Here, we have demonstrated that adenovirus-mediated overexpression of HCCS1 induces apoptosis in hepatocarcinoma cells and have further characterized the apoptotic cascade. The results showed that lysosomal cathepsin $D$ is released into the cytosol in response to HCCS1 overexpression and consequently triggers Bax insertion into the mitochondrial membrane, which leads to the release of cytochrome $c$. In addition, HCCS1 overexpression can induce an increase in intracellular free $\mathrm{Ca}^{2+}$ concentration, which also results in cytochrome $c$ release. The released cytochrome $c$ activates downstream caspases, leading to the occurrence of the late stages of apoptosis. Moreover, we demonstrated that the disruption of HCCS1 in mice leads to embryonic lethality, accompanied by abnormal labyrinth architecture resulting from the excessive proliferation of trophoblast cells in the placenta. These results suggest that $H C C S 1$ plays a role in apoptosis regulation and development.

Cell Death and Differentiation (2008) 15, 1481-1490; doi:10.1038/cdd.2008.73; published online 30 May 2008

Hepatocellular carcinoma suppressor 1 (HCCS1) has been identified as a novel tumor suppressor gene candidate via screening of the minimum region of high-frequency $\mathrm{LOH}$ on chromosome 17p13.3 and positional cloning in hepatocellular carcinoma in our previous study. ${ }^{1,2}$ Our recent studies demonstrated that replication-deficient adenovirus-mediated gene transfer of HCCS1 significantly inhibited the growth of human hepatocarcinoma cells in vitro or in vivo (submitted for publication), suggesting that HCCS1 may behave as a negative regulator of cell proliferation. However, the possible mechanism of this regulation is unclear. Since apoptosis is believed to play an important role in the maintenance of homeostasis and the inhibition of tumorigenesis, in the present study, we focused on the role of HCCS1 in the induction of apoptosis.

Contrary to the previous study, ${ }^{1}$ which highlighted HCCS1 as an important gene contributing to hepatocellular carcinoma tumorigenesis, another study regarding gene mutations and $\mathrm{LOH}$ suggested that the HCCS1 gene might be involved in the acceleration of the tumorigenic process and its mutation might not be the main mechanism of inactivation in the development of hepatocellular carcinoma. ${ }^{3}$ Although the authors came up with certain explanations for this discrepancy, further studies are required to investigate the role of HCCS1 in vivo.

Here, we provide definite experimental evidence demonstrating that adenovirus-mediated overexpression of HCCS1 promotes apoptosis of human hepatocarcinoma cells.
Furthermore, we have characterized the HCCS1-mediated apoptotic cascade. Moreover, we aimed to generate HCCS1 knockout mice to study the in vivo role of HCCS1 but found that the disruption of HCCS1 leads to an unexpected embryonic lethality. Further studies on the histology of the placenta from HCCS1 heterozygous intercrosses suggested that disruption of normal labyrinth architecture that resulted from the excessive proliferation of trophoblast cells in the $\mathrm{HCCS}^{-/-}$placenta may be the cause of embryonic lethality.

\section{Results}

HCCS1 overexpression induces apoptosis in hepatocarcinoma cells via cytochrome c/caspase-9/caspase-3 pathway. Our recent studies have demonstrated that infection with a replication-deficient recombinant adenovirus expressing HCCS1 (Ad-HCCS1) leads to the overexpression of HCCS1 in human hepatocarcinoma cells (Supplementary Figure S1a). Ad-HCCS1 exerted marked cytopathic effect against BEL7404 cells compared with Ad or Ad-EGFP, whereas the cytotoxic activity of Ad-EGFP was similar to that of Ad (Supplementary Figure S1b). To determine the possible contribution of apoptosis to Ad-HCCS1-mediated cytotoxicity, BEL7404 cells were infected with Ad-HCCS1 or Ad at a multiplicity of infection of 50 and assessed by fluorescent-activated cell sorting (FACS) following Annexin

\footnotetext{
${ }^{1}$ Laboratory for Cellular and Molecular Immunology, Shanghai Cancer Institute, Shanghai 200032, P.R. China and ${ }^{2}$ Shanghai Research Center for Model Organisms, Shanghai 201210, P.R. China

*Corresponding authors: XT Zhao, Laboratory for Cellular and Molecular Immunology, Shanghai Cancer Institute, Ln2200/25 Xie Tu Road, Shanghai 200032 , China. Tel/Fax: + 8621 64046360; E-mail: xtzhao@online.sh.cn and ZG Wang, Shanghai Research Center for Model Organisms, 3577 Jin-Ke Road, Zhang-Jiang Hi-Tech Park, Shanghai 201210, China. Tel/Fax: + 8621 58951591; E-mail: zhugangw@shsmu.edu.cn

Keywords: Hepatocellular carcinoma suppressor 1; apoptosis; calcium; cathepsin D; labyrinth abnormality

Abbreviations: HCCS1, Hepatocellular carcinoma suppressor 1; PI, prodiduim iodine; FACS, fluorescent-activated cell sorting; PARP, poly(ADP-ribose) polymerase; BIP, Bax-inhibiting peptide; PCNA, proliferation cell nuclear antigen; DMSO, dimethylsulfoxide

Received 04.1.08; revised 11.4.08; accepted 14.4.08; Edited by D Vaux; published online 30.5.08
} 
V-FITC/prodiduim iodine $(\mathrm{PI})$ staining. As shown in Figure 1a and b, Ad-HCCS1-infected BEL7404 cells exhibited a significant increase in Annexin $V$ staining $48 \mathrm{~h}$ post-infection $(81.6 \pm 3.1 \%$ in Ad-HCCS1-infected cells compared with $3.6 \pm 0.3 \%$ in untreated cells or $4.1 \pm 0.2 \%$ in Ad-infected cells). The nuclear morphological changes in response to Ad-HCCS1 infection of BEL7404 hepatocarcinoma cells were then examined by fluorescence microscopy after staining with Hoechst33258. Pyknotic and karyorrhectic nuclei appeared 72 and $96 \mathrm{~h}$ after Ad-HCCS1 infection (Supplementary Figure S1c). These results indicate that Ad-HCCS1 infection produces apoptosis in BEL7404 hepatocarcinoma cells. HCCS1-induced apoptosis was also supported by an increase in the nuclear staining observed after in situ TUNEL (terminal deoxynucleotidyltransferase (TdT)-mediated dUTP-biotin nick end-labeling) (Supplementary Figure S1d).

To evaluate the role of caspase in HCCS1-induced apoptosis, cleavages of several key components of the caspase family were detected by western blotting. As shown in Figure 1c, caspase- 3 and -9 , but not caspase- 8 and -12 were processed beginning at $48 \mathrm{~h}$ after Ad-HCCS1 infection. There was no cleavage of any procaspases detected even $96 \mathrm{~h}$ after Ad infection. We then examined the cleavage of the known caspase substrate poly(ADP-ribose) polymerase (PARP); as expected, it was observed to be cleaved after the infection (Figure 1c). To further discern the roles of caspases in HCCS1-induced apoptosis, we used the a

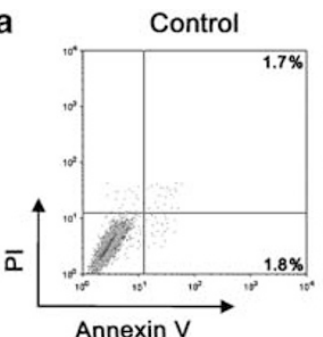

C

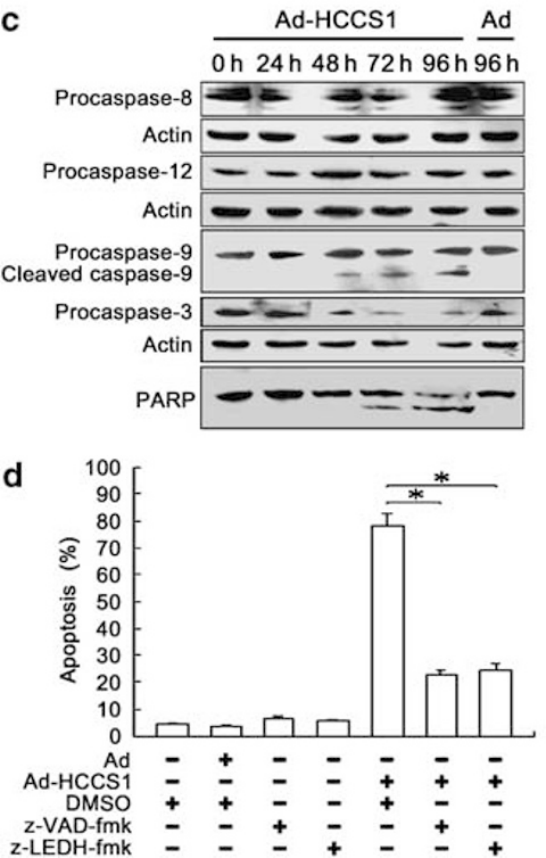

Ad

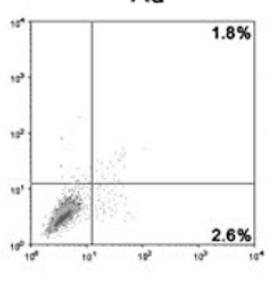

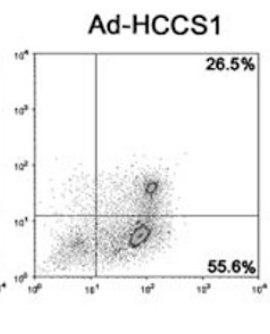

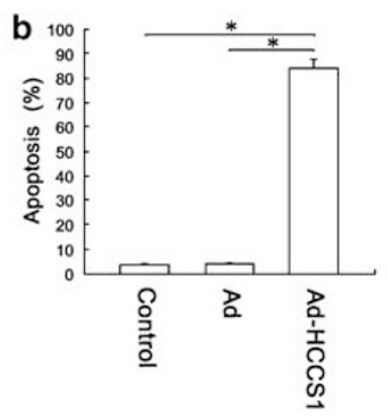

e

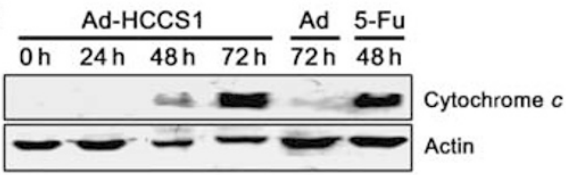

f
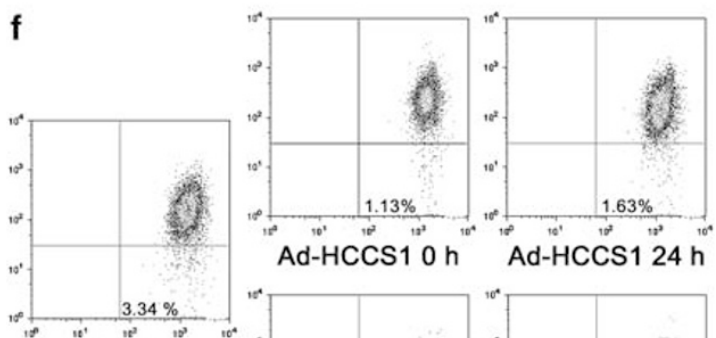

A
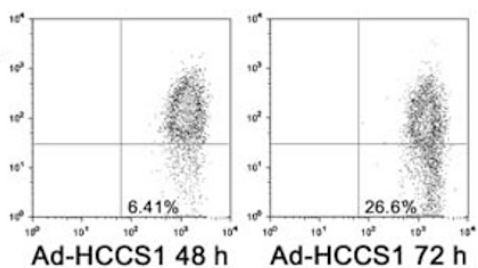

Figure 1 Ad-HCCS1 induces apoptosis of hepatocarcinoma cells by cytochrome c/caspase-9/caspase-3 pathway. (a) Untreated BEL7404 cells (left panel), or cells infected with Ad for $48 \mathrm{~h}$ (middle panel) or infected with Ad-HCCS1 for $48 \mathrm{~h}$ (right panel) were stained with Annexin V and PI, and analyzed by FACS. The dual parametric dot plots combining Annexin V-FITC and PI fluorescence show the viable cell population in the bottom left quadrant (Annexin $\mathrm{V}^{-} \mathrm{PI}^{-}$), the early apoptotic cells in the bottom right quadrant (Annexin $\mathrm{V}^{+} \mathrm{PI}^{-}$), and the late apoptotic cells in the top right quadrant (Annexin $\mathrm{V}^{+} \mathrm{PI}^{+}$). (b) Annexin $\mathrm{V}^{+} \mathrm{PI}^{-}$and Annexin $\mathrm{V}^{+} \mathrm{PI}^{+}$cells were assumed to be apoptotic cells. The apoptotic cells are indicated as a percentage of gated cells. Data represent means \pm S.D. from three independent experiments. Bars, S.D.; ${ }^{*} P<0.05$. (c) Western blot analyses of caspase-8, $-9,-12,-3$, and PARP. BEL7404 cells were infected with Ad or Ad-HCCS1. Whole cells were collected at $24 \mathrm{~h}$ intervals for $96 \mathrm{~h}$, and subjected to western blot analysis. (d) Inhibition of apoptosis by caspase inhibitors. BEL7404 cells were infected with Ad or Ad-HCCS1. After incubating for $48 \mathrm{~h}$ with z-VADfmk (pan-caspase inhibitor), or Z-LEDH-fmk (caspase-9 inhibitor), or solvent DMSO, cellular apoptosis was detected by Annexin V/PI staining. Data represent means \pm S.D. from three independent experiments. Bars, S.D.; ${ }^{*} P<0.05$. (e) Cytosolic fractions from infected cells were prepared and analyzed by western blot analyses with anticytochrome $c$ antibody. The cytosolic fraction from 5-Fu-treated cells was used as the positive control. (f) Measurement of mitochondrial membrane potential. Infected cells were collected and stained with JC-1, and then analyzed by FACS. Cells with normal polarized mitochondrial membranes emit green-orange fluorescence (top right quadrant). The number in the bottom right quadrant of each dot plot represents the percentage of cells that emit only green fluorescence attributable to depolarized mitochondrial membranes 
pan-caspase inhibitor z-VAD-fmk and a caspase-9 inhibitor z-LEDH-fmk. As shown in Figure 1d, both inhibitors provided significant protection from HCCS1-induced apoptosis.

To test whether cytochrome $c$ is released from mitochondria during HCCS1-induced apoptosis, its cytosolic translocation was examined by western blot. As shown in Figure 1e, cytochrome $c$ in the cytosol of Ad-HCCS1 infected cells was observed at $48 \mathrm{~h}$ after infection, and its levels significantly increased at $72 \mathrm{~h}$. To see whether the release of cytochrome $c$ is a consequence of mitochondrial membrane potential $\left(\Delta \psi_{\mathrm{m}}\right)$ loss, BEL7404 cells were infected with Ad or Ad-HCCS1, and then stained with $\mathrm{JC}-1$, which reflects changes in $\Delta \psi_{\mathrm{m}}$ as a fluorescence shift from orange to green, and analyzed by FACS. As shown in Figure 1f, there is a clear increase in the percentage of cells (lower right quadrants) that emitted only green fluorescence (representing cells with depolarized mitochondrial membranes) $72 \mathrm{~h}$ after Ad-HCCS1 infection. This observation supports the previous finding ${ }^{4}$ that the release of cytochrome $c$ can precede the loss of mitochondrial membrane potential in some apoptotic pathway.

HCCS1 overexpression induces activation of Bax that is not associated with Bid cleavage. Bax can fully integrate into the mitochondrial membrane and then facilitate the release of cytochrome $c$. $^{5,6}$ Therefore, we investigated the subcellular distribution of Bax by western blot. Bax protein was not only found in the cytosol but also observed to be loosely associated with mitochondria in normal cells, and the mitochondrial membrane insertion of Bax was shown by its resistance to alkaline extraction similar to the mitochondrial protein Cox II. As shown in Figure 2a, Bax was inserted into the mitochondrial membrane of Ad-HCCS1-infected cells $36 \mathrm{~h}$ post-infection. Although mitochondrial Bax was also examined at an earlier time point, it could not be observed at the time point of $24 \mathrm{~h}$ post-infection (data not shown). The original p21 Bax was partially cleaved to p18 Bax, which has been demonstrated to accelerate the apoptotic process. ${ }^{7}$ p18 Bax was observed at $60 \mathrm{~h}$ post-infection in the alkalineresistant fraction; however, the cleavage of Bax was not detected in the cytosol of Ad-HCCS1-infected cells (Figure 2a). We then used the Bax-inhibiting peptide (BIP) V5, which inhibits Bax-mediated translocation of cytochrome $c$ and suppresses mitochondria-dependent apoptosis. ${ }^{8}$ As shown in Figure 2b, BIP V5 could effectively suppress apoptosis induced by Ad-HCCS1 infection.

Was Bid activated (tBid produced) and translocated to mitochondria together with Bax? In Ad-HCCS1-infected cells, Bid remained entirely confined to the cytosol as a full-length p22 protein (Figure $2 \mathrm{c}$ ). The results indicate that HCCS1 overexpression induces Bax insertion into the mitochondrial membrane that is not associated with the activation of Bid, and then initiates the apoptosis.

Intracellular $\mathrm{Ca}^{2+}$ is involved in HCCS1-induced apoptosis. To test whether an increase in intracellular $\mathrm{Ca}^{2+}$ plays a role in HCCS1-induced apoptosis, the level of intracellular free $\mathrm{Ca}^{2+}$ concentration $\left(\left[\mathrm{Ca}^{2+}\right]_{\mathrm{i}}\right)$ was analyzed by using Flou-3/AM, a cell-permeable $\mathrm{Ca}^{2+}$ indicator. The infection of BEL7404 cells with Ad-HCCS1 resulted in an increase of $\left[\mathrm{Ca}^{2+}\right]_{i}$ in a time-dependent
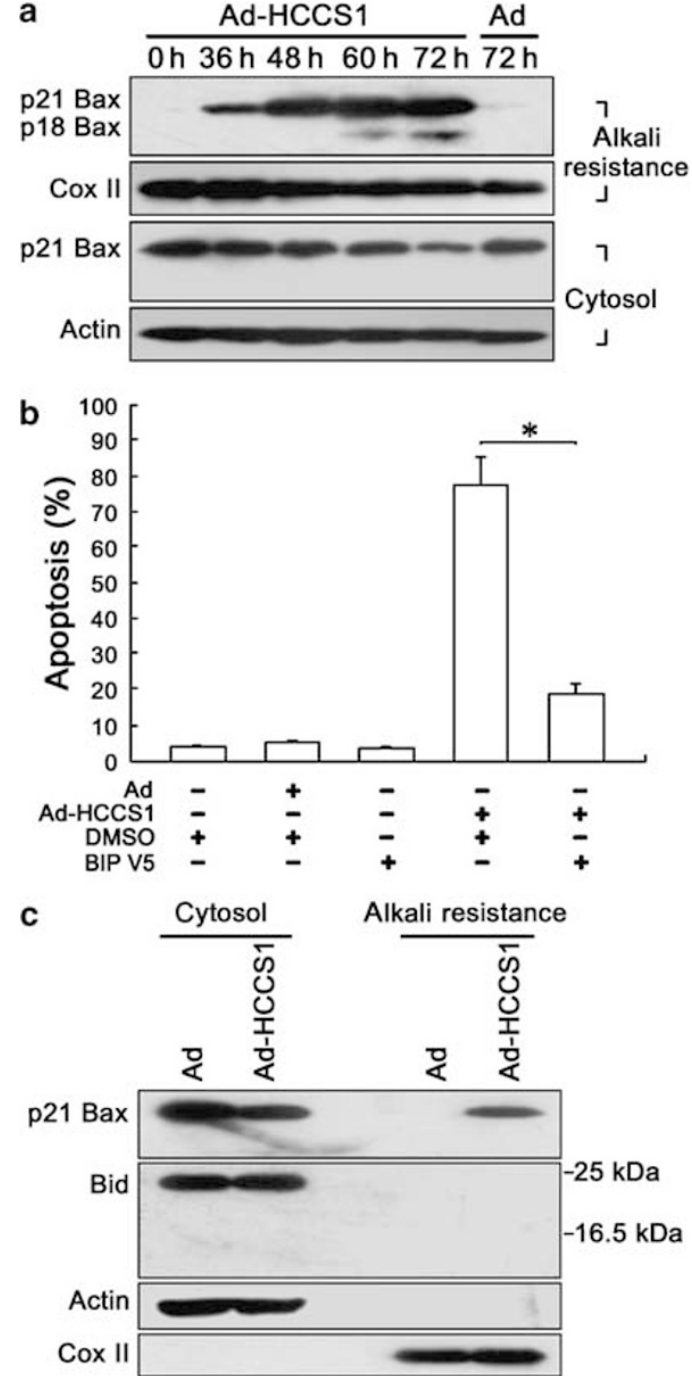

Figure 2 Ad-HCCS1 triggers Bax insertion into the mitochondrial membrane. (a) BEL7404 cells were infected with Ad or Ad-HCCS1. After incubation for the various times indicated, cytosolic and mitochondrial fractions were prepared. Mitochondrial fractions were then treated with $\mathrm{Na}_{2} \mathrm{CO}_{3}$ to produce alkali-resistant fractions. Both cytosolic and alkali-resistant fractions were analyzed by western blotting for the presence of Bax and Cox II. (b) Inhibition of apoptosis by BIP V5. BEL7404 cells were infected with Ad or Ad-HCCS1 and incubated for $48 \mathrm{~h}$ in the presence of BIP V5 or DMSO. Apoptosis was analyzed by Annexin V/PI staining. Data represent means \pm S.D. from three independent experiments. Bars, S.D.; ${ }^{\star} P<0.05$. (c) Bid remains as a full-length protein in the cytosol. BEL7404 cells were infected with Ad or Ad-HCCS1 for $48 \mathrm{~h}$ and Bax, Bid, Cox II, and actin in the cytosolic and alkali-resistant fractions were analyzed by western blotting

manner, shown as stronger fluorescent intensities compared with the cells infected with Ad (Figure $3 a$ and b). Then, we investigated the effect of Bapta-AM, an intracellular $\mathrm{Ca}^{2+}$ chelator, on HCCS1-induced apoptosis; the results demonstrated that Bapta-AM alleviated apoptosis induced by Ad-HCCS1 infection in a dose-dependent manner (Figure 3c). Our results suggest that changes in intracellular $\mathrm{Ca}^{2+}$ homeostasis appear to be involved in HCCS1-induced apoptosis. 
a

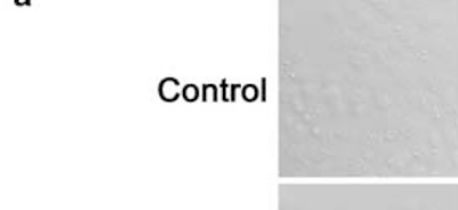

Ad

$12 \mathrm{~h}$ after infection

Ad-HCCS1

$12 \mathrm{~h}$ after infection

Ad

$24 \mathrm{~h}$ after infection

\section{Ad-HCCS1}

$24 \mathrm{~h}$ after infection

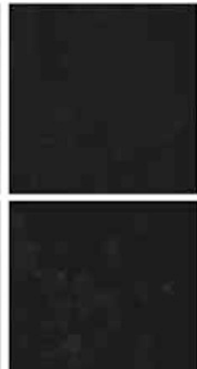

b

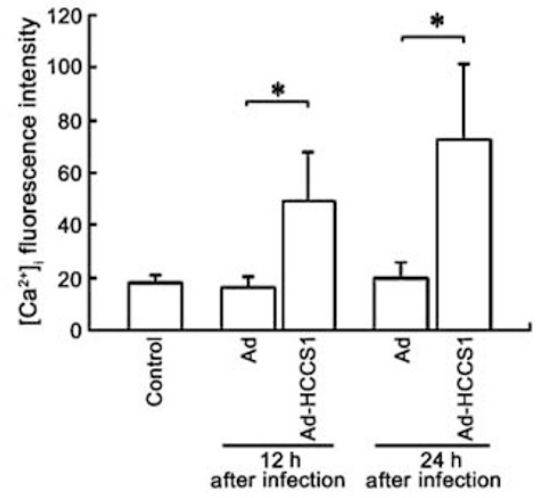

c

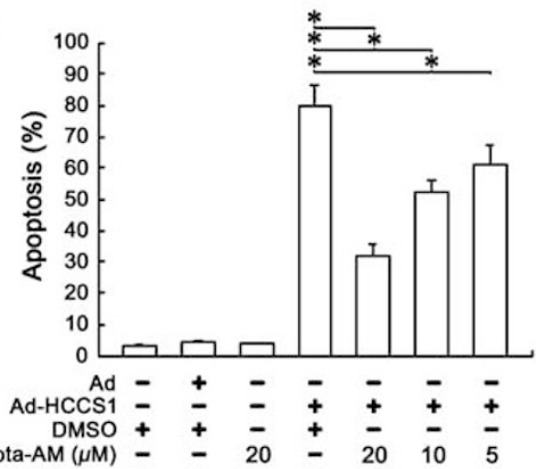

Figure 3 Intracellular $\mathrm{Ca}^{2+}$ is involved in HCCS1-induced apoptosis. (a) Analysis of $\left[\mathrm{Ca}^{2+}\right]$. BEL7404 cells were infected with Ad or Ad-HCCS1 for 12 or $24 \mathrm{~h}$, respectively. The level of $\left[\mathrm{Ca}^{2+}\right]_{\mathrm{i}}$ in infected or uninfected cells was evaluated by confocal laser scanning microscopy with the fluorescence probe Fluo-3/AM. Stronger $\left[\mathrm{Ca}{ }^{2+}\right]$ fluorescence was visualized following Ad-HCCS1 infection (original magnification, $\times 1000$ ). (b) The data represent the means of cellular [ $\left.\mathrm{Ca}^{2+}\right]_{i}$ fluorescence intensity. The bars above the column show the S.D. calculated from 100 cells; ${ }^{*} P<0.05$. (c) Inhibition of apoptosis by Bapta-AM (a calcium chelator). BEL7404 cells were infected with Ad or Ad-HCCS1 and incubated for $48 \mathrm{~h}$ in the presence of DMSO or Bapta-AM at the different concentrations indicated. Apoptosis was detected by Annexin V/PI staining. Data represent means \pm S.D. from three independent experiments. Bars, S.D.; ${ }^{*} P<0.05$

\begin{abstract}
Lysosomal cathepsin D is involved in HCCS1-induced apoptosis. We further investigated the lysosomal permeabilization and the subcellular distribution of cathepsin D in the cells following Ad-HCCS1 infection. Ad-HCCS1 infection initiated an early lysosomal rupture because LysoTrack Red, an acidic organelle-specific probe, could not be accumulated in the granular cellular structures of the cells $24 \mathrm{~h}$ after infection (Figure $4 \mathrm{a}$ ). Moreover, cathepsin D was also detected in the cytosolic fraction at the same time (Figure $4 b$ ), indicating its release from the lysosome. To investigate whether cathepsin $D$ is indeed involved in HCCS1-induced apoptosis, Ad-HCCS1-infected cells were cultured in the presence of pepstatin A (an aspartic protease inhibitor that inhibits cathepsin $D$ ) and analyzed for apoptosis. As shown in Figure 4c, pepstatin A could alleviate apoptosis in a dose-dependent manner, suggesting that cathepsin D may play a role in HCCS1induced apoptosis.
\end{abstract}

Cathepsin $\mathrm{D}$ and $\mathrm{Ca}^{2+}$ trigger the release of cytochrome $c$ in HCCS1-induced apoptosis. Since it was demonstrated that Ad-HCCS1 induced the cytosolic translocation of cathepsin $\mathrm{D}$ and the elevation of $\left[\mathrm{Ca}^{2+}\right]_{i}$ prior to the translocation of Bax and cytochrome $c$, we postulated that they both initiate the mitochondrial apoptotic signal. To confirm this hypothesis, the translocations of cytochrome $c$ and Bax in infected cells were analyzed after incubating them for $48 \mathrm{~h}$ with pepstatin A or Bapta-AM. Both the inhibitors could block the release of cytochrome $c$ into the cytosol, but only pepstatin A could inhibit Bax insertion into the mitochondrial membrane (Figure 4d). It is implied that cathepsin $\mathrm{D}$ triggers Bax activation and then causes the release of cytochrome $c$ in HCCS1-induced apoptosis, whereas $\mathrm{Ca}^{2+}$-induced cytochrome $c$ release is not associated with Bax translocation.

Targeted disruption of HCCS1 leads to embryonic lethality. To elucidate the role of HCCS1 in vivo, we generated HCCS1-deficient mice by gene targeting in ES cells. We replaced exons 1 and 2 with a neomycin resistance $\left(n e o^{r}\right)$ gene (Figure 5a) and identified the targeted ES cell clones with Southern blot analysis using probes external to the targeting construct (Figure 5b). Targeted ES cell clones contributed to the germ line of chimeric mice and generated HCCS1 heterozygous null mice $\left(\mathrm{HCCS}^{+/-}\right)$. Heterozygous male and female mice displayed no overt phenotype and were fertile. However, we were unable to identify a single HCCS1 homozygous mouse $\left(\mathrm{HCCS}^{-/-}\right)$among the 111 offspring born from the crosses between HCCS1 heterozygous mice. The surviving pups were either $\mathrm{HCCS}^{+/+}$(33 of 111) or HCCS1 ${ }^{+/-}$(78 of 111). These results represent the Mendelian ratio of mutant and wild-type 


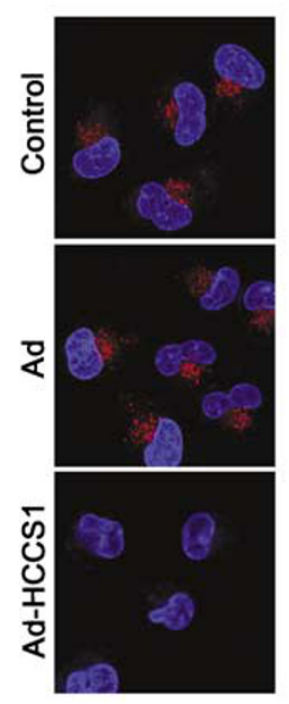

b

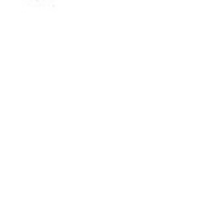

Cathepsin D

Cytosol $\begin{gathered}\text { Whole } \\ \text { cell }\end{gathered}$

Actin
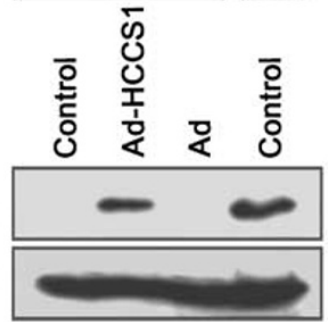

c

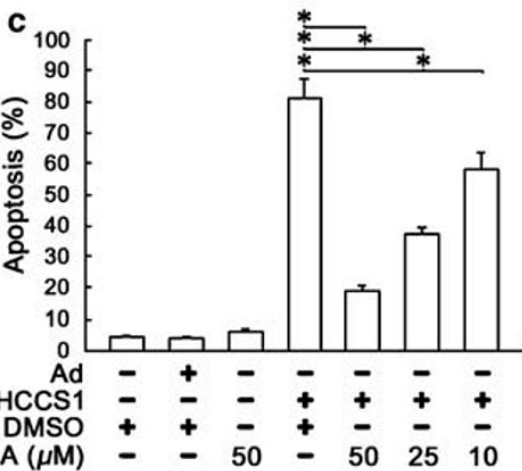

d

$$
\underline{\text { Ad }}
$$

\section{Ad-HCCS1}
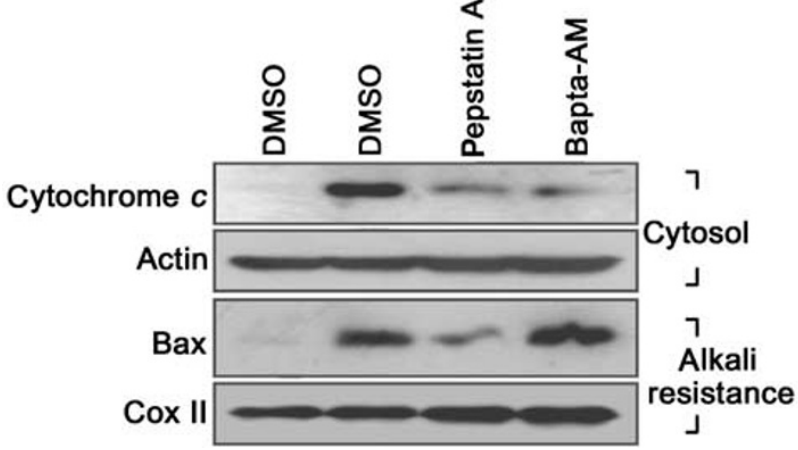

Figure 4 Lysosomal efflux of cathepsin D is committed to HCCS1-induced apoptosis by triggering Bax integration into mitochondrial membrane and cytochrome $c$ release, whereas intracellular $\mathrm{Ca}^{2+}$-induced cytochrome $c$ release is not associated with Bax activation. (a) BEL7404 cells were infected with Ad or AdHCCS1 for $24 \mathrm{~h}$. Uninfected and infected cells were then loaded with LysoTrack Red and nuclei were stained with DAPI (blue). Lysosomal stability assessment was performed by confocal microscopy. LysoTrack Red uptake was completely prevented in the cells infected with Ad-HCCS1. (b) Cytosolic fractions from uninfected BEL7404 cells or the cells infected with Ad or Ad-HCCS1 for $24 \mathrm{~h}$ were prepared and analyzed by western blotting with anti-cathepsin $D$ antibody. (c) Inhibition of apoptosis by pepstatin A. BEL7404 cells were infected with Ad or AdHCCS 1 for $48 \mathrm{~h}$ in the presence of solvent DMSO or pepstatin A at the different concentrations indicated. Apoptosis was detected by Annexin V/PI staining. Data represent means \pm S.D. from three independent experiments. Bars, S.D.; ${ }^{*} P<0.05$. (d) BEL7404 cells were infected with Ad or Ad-HCCS1 and incubated with pepstatin A, Bapta-AM, or DMSO. Cytosolic fractions were prepared at $48 \mathrm{~h}$ after infection, and cytochrome $c$ in the cytosolic fraction was analyzed by western blotting with an anti-cytochrome $c$ antibody (top panel). Mitochondrial fractions were prepared at $36 \mathrm{~h}$ after infection and treated with $\mathrm{Na}_{2} \mathrm{CO}_{3}$ to produce alkali-resistant fractions, which were then analyzed by western blotting for the presence of $\mathrm{Bax}$ and Cox II (bottom panel)

alleles when HCCS1 deficiency results in embryonic death. To determine the timing of this lethality, we isolated and genotyped embryos (Figure $5 \mathrm{c}$ ) at various gestational days. It was found that $\mathrm{HCCS}^{-/-}$embryos died before embryonic day (E) 12.5 and were small and developmentally retarded compared with their littermate controls beginning on E7.5. The comparison between $\mathrm{HCCS}^{-/-}$and $\mathrm{HCCS}^{+/+}$ embryos at E9.5 is shown in Figure $5 \mathrm{~d}$.

The embryonic lethality of HCCS1-null mice possibly due to abnormal labyrinth architecture in the placenta. To test whether there is any defect in the $\mathrm{HCCS}^{-/-}$placenta, a histological examination of the placenta from the HCCS1 heterozygous intercrosses was performed. Hematoxylin- and eosin-stained sections demonstrated that the wild-type labyrinth had a porous appearance-with trophoblast cells being well organized and distributed around the fetal and maternal blood vessels at E10.5 - that facilitated the exchange of oxygen and nutrients between the mother and the fetus. However, the HCCS1 mutant labyrinth layer was severely disrupted with an accumulation of large clusters of densely packed trophoblast cells at the expense of maternal and fetal blood spaces (Figure 6a and b). The differences between HCCS1 mutant and wild-type placentae was observed at E9.5 (data not shown). In the wild-type placenta, trophoblast cells lining the maternal blood spaces appeared elongated and thin and expressed endogenous alkaline phosphatase activity at the brush border. Therefore, we further characterized the decrease in maternal and fetal blood spaces by analyzing the alkaline phosphatase-positive cells, which reflect the trophoblast transport surface area within the labyrinth. It was shown that the trophoblast transport surface area within the HCCS1-deficient placental labyrinth was significantly reduced, indicating a considerably less extensive villous structure (Figure 6c). Furthermore, we measured cellular proliferation in $\mathrm{HCCS}^{+/+}$and $\mathrm{HCCS}^{-1-}$ placentae by proliferation cell nuclear antigen (PCNA) immunohistochemical staining. Analysis of the labyrinth from E10.5 placentae indicated that $\mathrm{HCCS}^{-1-}$ trophoblast cells had a markedly increased expression of PCNA than wild-type littermate controls (Figure 6d). Taken together, our results suggest that the embryonic lethality of $\mathrm{HCCS}^{-/-}$ mice probably results from the excessive proliferation of trophoblast cells, which disrupts the normal labyrinth architecture and decreases vascularization in the placenta.

\section{Discussion}

In this study, we identified the role of apoptosis in HCCS1 overexpression-mediated cytotoxicity and further investigated the possible molecular mechanism of HCCS1-induced apoptosis. It was shown that Ad-HCCS1 infection induces the lysosomal disintegration and the release of cathepsin $D$ to the cytosol. Released cathepsin $\mathrm{D}$ leads to the mitochondrial translocation of Bax and the release of cytochrome $c$ followed by the activation of caspase- 9 and -3 . The release of cytochrome $c$ also results from the elevation of $\left[\mathrm{Ca}^{2+}\right]_{\mathrm{i}}$ initiated by Ad-HCCS1 infection. Our results propose a novel cathepsin $\mathrm{D} / \mathrm{Bax} /$ cytochrome c/caspase pathway and a $\mathrm{Ca}^{2+} /$ cytochrome c/caspase pathway in HCCS1-induced apoptosis (Figure 7). 

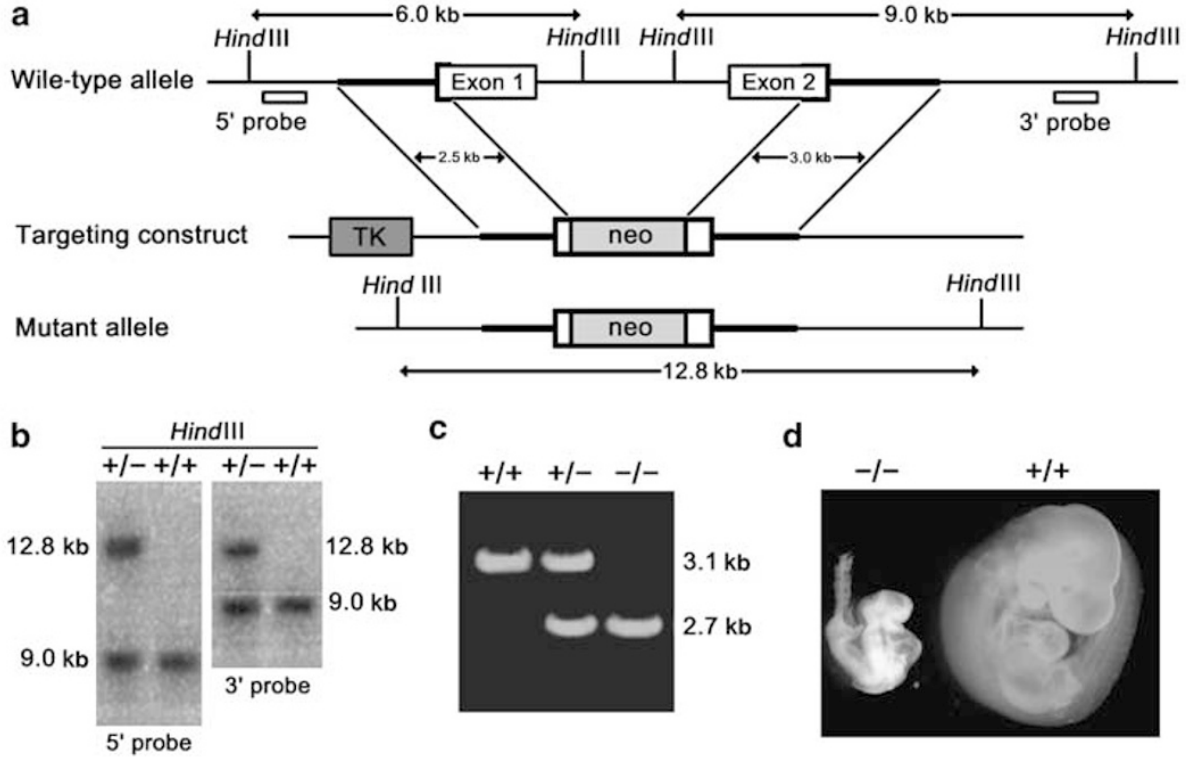

C

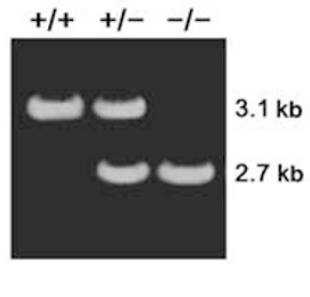

d

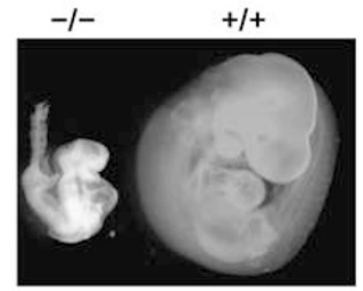

Figure 5 Targeted disruption of HCCS1 leads to embryonic lethality. (a) A targeting strategy used for the inactivation of the murine HCCS1 gene is shown. The targeting construct contained an $\sim 5.5 \mathrm{~kb}$ genomic sequence of $H C C S 1$ with a neo cassette. A homologous recombination within the HCCS1 locus introduces the neo gene and deletes intron 1 and part of exon 1 and 2. (b) Southern blot analysis of genomic DNA isolated from target ES cell clones. The band of $12.8 \mathrm{~kb}$ corresponds to the target allele. (c) Genotyping of HCCS1 embryos with PCR. The wild-type HCCS1 allele was detected using primers that amplified a fragment of $3.1 \mathrm{~kb}$, and a fragment of $2.7 \mathrm{~kb}$ was amplified from the targeted allele. (d) Whole-mount preparations of embryos from $\mathrm{HCCS} 1^{+/}$intercrosses at E9.5. The $\mathrm{HCCS}^{-/-}$embryos were small and displayed abnormal development

Lysosomal cathepsins have been shown to be readily translocated to the cytosol and to mediate apoptosis in response to various stimuli. ${ }^{9}$ Further, released cathepsin $D$, an aspartic protease, has been reported to trigger Bax activation, which is not associated with Bid activation and result in selective AIF release. ${ }^{10}$ This pathway was partially supported by our results suggesting that released cathepsin D triggers the insertion of Bax into mitochondrial membranes without the cleavage of Bid. The release of cathepsin D following lysosomal permeabilization constitutes an initial step in the HCCS1-induced apoptotic cascade. Since cathepsin D has been demonstrated to remain active at a neutral $\mathrm{pH},{ }^{10}$ extralysosomal cathepsin D in Ad-HCCS1-infected cells might activate Bax by degrading cytosolic chaperones, including certain isoforms of cytosolic 14-3-3 proteins and the Ku70 protein, which sequester Bax in an inactive conformation in healthy cells and undergo dissociation during apoptosis. ${ }^{11,12}$

Inconsistent with the previous pathway, ${ }^{10}$ our experiments highlighted that the release of cytochrome $c$, but not AIF, is the central event of apoptotic cascades in response to HCCS1 overexpression. AIF can cause apoptotic cell death once it translocates from the mitochondria to the nucleus. ${ }^{13}$ Although AIFs were released from mitochondria following Ad-HCCS1 infection, the nuclear translocation of AIF was not detected even when HCCS1-induced pyknosis was observed. And the AIF inhibitor $N$-phenylmaleimide ${ }^{14}$ could not protect cells from HCCS1-induced apoptosis (Supplementary Figure S2). These results suggest that AIF is not involved in HCCS1induced apoptosis. We also measured the $\Delta \psi_{\mathrm{m}}$ changes of the cells infected with Ad-HCCS1. It remains uncertain whether the loss of $\Delta \psi_{\mathrm{m}}$ occurs as an initiator, or an effect of apoptosis, or is actually necessary of apoptosis induction to occur. Although the dissipation of $\Delta \psi_{\mathrm{m}}$ was shown to be a requirement for cytochrome $c$ release from the mitochondria, ${ }^{15,16}$ other studies ${ }^{17-19}$ suggest that the loss of $\Delta \psi_{m}$ occurs secondary to cytochrome $c$ release and that $\mathrm{Bax}$ participation may be a more plausible candidate for cytochrome $c$ release than $\Delta \psi_{\mathrm{m}}$. In our system, it was showed that HCCS1 overexpression induced cytochrome $c$ release following Bax translocation but without obvious depolarized mitochondrial membranes. Since the disruption of $\Delta \psi_{\mathrm{m}}$ may appear to be responsible for the release of AIF into the cytosol, ${ }^{17}$ one can conceive that the loss of $\Delta \psi_{\mathrm{m}}$ followed by AIF release may be a subsequent event in the HCCS1induced apoptotic pathway.

Elevation of $\left[\mathrm{Ca}^{2+}\right]_{i}$ can trigger the release of mitochondrial cytochrome $c$ and the activation of caspases. ${ }^{20}$ The $\mathrm{Ca}^{2+}$. induced apoptotic cascade may be associated with activated $\mathrm{Ca}^{2+}$-sensitive enzymes. Calpain, a calcium-dependent cysteine protease, has been shown to be associated with $\mathrm{Ca}^{2+}$-induced apoptosis. ${ }^{21}$ Although chelation of intracellular $\mathrm{Ca}^{2+}$ did not prevent staurosporine-induced Bax translocation to mitochondria, ${ }^{22}$ it was reported that calpain could cleave Bax to p18 Bax to enhance Bax-induced apoptosis. ${ }^{7,23}$ Therefore, we measured the $\left[\mathrm{Ca}^{2+}\right]_{i}$ of Ad-HCCS1-infected cells to investigate its role in HCCS1-induced apoptosis. The results showed that HCCS1 overexpression led to an early increase of $\left[\mathrm{Ca}^{2+}\right]_{i}$. Buffering of cytosolic $\mathrm{Ca}^{2+}$ by Bapta-AM could block the release of cytochrome $c$ and alleviate apoptosis following Ad-HCCS1 infection, but it had no effect on the translocation of Bax or on its cleavage (Supplementary Figure S3c). This was supported by the observation that the 

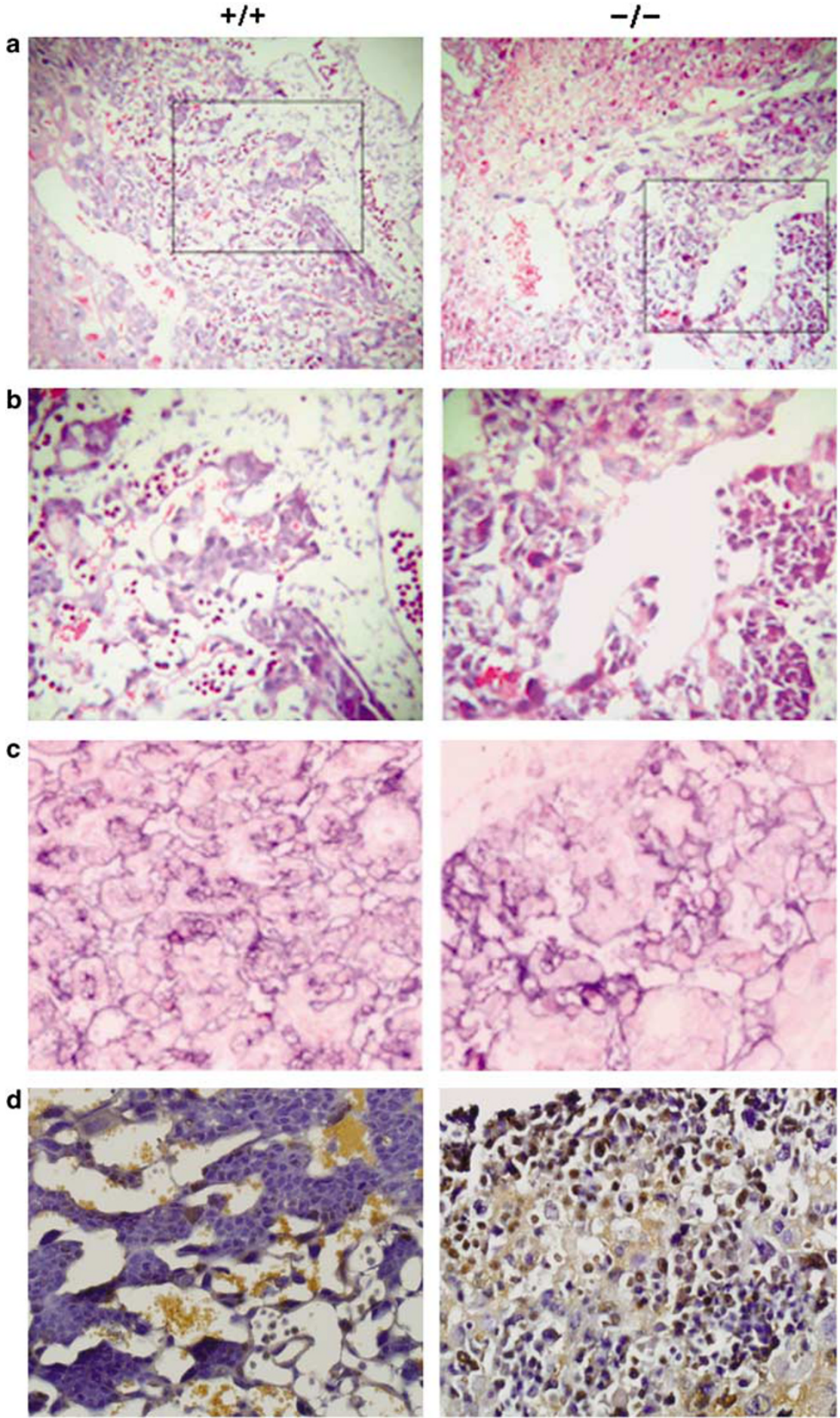

Figure $6 \mathrm{HCCS}^{-/-}$mice show placental dysplasia in the labyrinth layer. $(\mathbf{a}, \mathbf{b})$ Hematoxylin and eosin staining of placental sections from E10.5 $\mathrm{HCCS} 1^{+/+}$and $\mathrm{HCCS} 1^{-/-}$ mice. The boxed areas in a are shown at a higher magnification in b. (c) Analysis of alkaline phosphatase-positive cells in $H C C S 1^{+/+}$and $H C C S 1^{-/-}$placentae at E10.5. (d) Immunohistochemical staining for PCNA in $\mathrm{HCCS}^{+/+}$and $\mathrm{HCCS}^{-/-}$placentae at E10.5. Original magnification: $\times 200(\mathbf{a}), \times 400(\mathbf{b}, \mathbf{c}, \mathbf{d})$

activity of calpain was not increased in Ad-HCCS1-infected cells and that the calpain inhibitor could not inhibit the generation of p18 Bax and HCCS1-induced apoptosis (Supplementary Figure S3). Our results indicate that elevation of $\left[\mathrm{Ca}^{2+}\right]_{\mathrm{i}}$ induced by $\mathrm{HCCS} 1$ overexpression stimulates the release of cytochrome $c$, which is not associated with Bax translocation, and consequently leads to cell apoptosis. HCCS1 overexpression induces Bax cleavage by an unknown pathway, which is not mediated by calpain. The detailed elucidation of this pathway awaits further investigations.

On the other hand, we generated knockout mice to investigate the in vivo role of HCCS1 in tumorigenesis. 


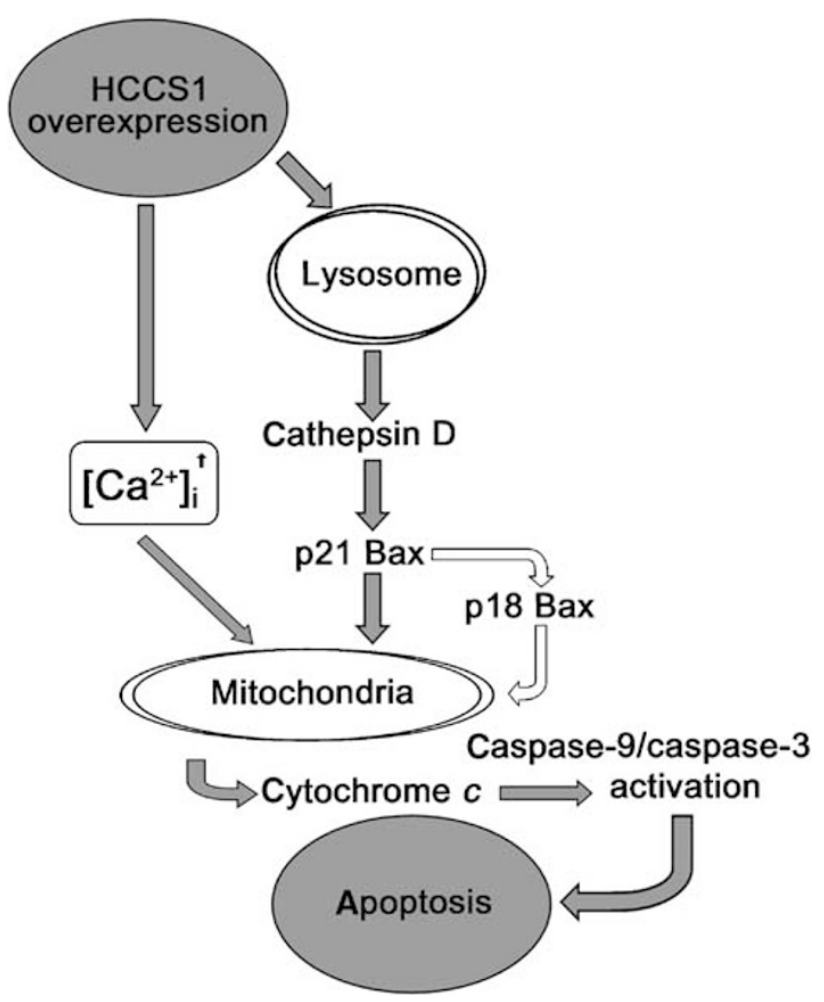

Figure 7 Proposed mechanisms of HCCS1-induced apoptosis. In this model, HCCS1 overexpression causes the lysosomal efflux of cathepsin D, which then induces cytosolic Bax insertion into the mitochondrial membrane followed by cytochrome $c$ release from mitochondria. Mitochondrial Bax is cleaved partially to produce $\mathrm{p} 18 \mathrm{Bax}$, which can accelerate the apoptotic process. Meanwhile, HCCS1 overexpression also causes $\left[\mathrm{Ca}^{2+}\right]_{i}$ elevation, which in turn leads to cytochrome $c$ release. Released cytochrome $c$ induces the activation of downstream caspases and late stages of apoptosis

However, HCCS1 mutant embryos unexpectedly suffered death by E12.5. Survival and growth of the fetus are still critically dependent on the placenta. Inactivation of various genes, including several tumor suppressor genes, leads to embryonic lethality as a result of abnormal placental development. ${ }^{24}$ For example, it was demonstrated that inactivation of the retinoblastoma $(R b)$ gene leads to embryonic lethality. ${ }^{25}$ Further investigations on $R b$-deficient placentae showed that the loss of $\mathrm{Rb}$ leads to excessive proliferation of trophoblast cells and a severe disruption of the normal labyrinth architecture in the placenta. The defect in $R b$-deficient placentae results in a decreased surface area required for oxygen/nutrient exchange between the mother and the fetus. A similar alteration of the cellular architecture of the labyrinth was observed in HCCS1-deficient placentae, indicating that a defect in proliferation in $\mathrm{HCCS}^{-/-}$placental trophoblast cells may be the lethal cause of embryonic death in $\mathrm{HCCS}^{-1-}$ mice. However, heterozygous HCCS1 mice had lived beyond 12 months without evidence of significant disease (data not shown), indicating that HCCS1 haploinsufficiency may not be a critical feature for normal HCCS1 function. Further analysis of HCCS1 homozygous mice, such as rescuing developing embryos from placental failure, should provide us with a better understanding of the role of HCCS 1 in development and tumorigenesis.
Notably, HCCS1 is demonstrated to be a homologue of yeast $V p s 53$ by cDNA sequence alignment. In yeast, Vps53 is a subunit of the Golgi-associated retrograde protein complex, which is involved in retrograde transport from the early and late endosome to the late Golgi. ${ }^{26}$ Human Vps53 or HCCS1 was shown to have analogous characteristics to the yeast homologue and co-localized with mannose-6-phosphate receptor, ${ }^{27}$ which is the primary receptor for the lysosomal aspartyl protease cathepsin D. ${ }^{28}$ Based on these observations, one may conceive that overexpressed HCCS1 induces the efflux of cathepsin $D$ by executing an abnormal transport function in the endosomal/lysosomal system. However, the mechanism by which overexpressed HCCS1 induces the elevation of intracellular calcium is not yet clear. Studies to elucidate these mechanisms are in progress.

To our knowledge, few transport proteins have been demonstrated to play a role in apoptosis regulation or development. In this study, we have proved the induction of apoptosis by HCCS1 overexpression and have characterized the HCCS1-induced apoptotic cascade. Meanwhile, data from the present investigation implied a non-cell autonomous role of HCCS1 during placental development. Further research into the roles of HCCS1 as a transport protein and as a regulator of apoptosis may lead to new strategies for controlling the development and growth of tumors.

\section{Materials and Methods}

Cell lines and culture conditions. The human hepatoma cell line BEL7404 and SMMC-7721 was purchased from Shanghai Cell Collection (Chinese Academy of Science, Shanghai, China), and cultured at $37^{\circ} \mathrm{C}$ in Dulbecco's modified Eagle's medium (HyClone, Logan, UT, USA) containing $10 \%$ fetal bovine serum. The intracellular calcium chelator, Bapta-AM, was purchased from BioMol (Plymouth Meeting, PA, USA) and used at final concentrations of $50 \mu \mathrm{M}$. The aspartic protease inhibitor, pepstatin A, was a product of Santa Cruz Biotechnology Inc. (Santa Cruz, CA, USA) and was used at a final concentration of $50 \mu \mathrm{M}$. The irreversible pan-caspase inhibitor, z-VAD-fmk, and the caspase-9 inhibitor, z-LEDHfmk, were purchased from R\&D systems (Minneapolis, MN, USA), and both were used at final concentrations of $50 \mu \mathrm{M}$. BIP V5 was purchased from Calbiochem (La Jolla, CA, USA) and used at a final concentration of $200 \mu \mathrm{M}$. Dimethylsulfoxide (DMSO; Sigma-Aldrich Co., St. Louis, MO, USA) was used at a corresponding dilution as a solvent control.

Generation of adenoviral vectors and infection procedure. The HCCS1 CDNA was obtained as described previously. ${ }^{1} \mathrm{Ad}-\mathrm{HCCS} 1$, a replicationdeficient adenovirus that expresses HCCS1, and Ad-EGFP were constructed in adherence with the technical protocol of the AdEasy vector system (Strategene, La Jolla, CA, USA). Ad is a control recombinant adenovirus that does not carry any transgene but has the same adenovirus backbone as Ad-HCCS1. The presence of the transgene in the finally isolated viral stock was confirmed by PCR. The recombinant adenoviruses were titrated by a plaque assay. Infection with $\mathrm{Ad}$ HCCS1 and Ad was accomplished by incubating the cells at a multiplicity of infection of 50 in serum-free medium for $2 \mathrm{~h}$, followed by addition of the medium containing $10 \%$ serum and further incubation for the required time at $37^{\circ} \mathrm{C}$.

Analysis of apoptosis by Annexin V/PI staining. Infected cells were washed, resuspended in the staining buffer, and examined with Annexin V-FITC Apoptosis Detection kit (KeyGen Biotech Co., Nanjing, China) according to the manufacturer's instructions. Stained cells were analyzed by FACS (FACScalibur; Becton Dickinson, Mountain View, CA, USA).

Subcellular fractionation and western blot analyses. Whole-cell lysates and cytosolic and mitochondrial fractions were prepared and subjected to western blot analysis as described previously. ${ }^{29}$ Blots were probed with anticaspase-3, anti-actin, anti-AIF, anti-cathepsin D (Santa Cruz Biotechnology), 
anti-PARP, anti-caspase-9, anti-Bid, anti-Bax (Cell Signaling Technology, Beverly, MA, USA), anti-caspase-8 (Chemicon, Temecula, CA, USA), and anti-caspase-12 (Calbiochem)

Alkaline extraction. The mitochondrial fractions from infected cells were resuspended in $0.1 \mathrm{M} \mathrm{Na}_{2} \mathrm{CO}_{3}(\mathrm{pH} 11.5)$, incubated for 30 min on ice, and then centrifuged $(75000 \times g, 20 \mathrm{~min})$. The pellet was analyzed by western blotting as an alkali-resistant fraction.

Analysis of $\Delta \psi_{\mathrm{m}}$. Infected cells were stained with the fluorochrome JC-1 $\left(1.25 \mu \mathrm{g} / \mathrm{ml}\right.$; Molecular Probes) according to the previous study. ${ }^{30}$ The membrane potential was measured by using FACS. JC-1 aggregates were detected at $585 \mathrm{~nm}$ and JC-1 monomers at $530 \mathrm{~nm}$.

Analysis of $\left[\mathrm{Ca}^{2+}\right]_{i}$. Analysis of $\left[\mathrm{Ca}^{2+}\right]_{\mathrm{i}}$ was performed by using an intracellular free $\mathrm{Ca}^{2+}$ fluorescent probe, Flou-3/AM (Molecular Probes), as described previously. ${ }^{31}$ Flou-3/AM-loaded cells were examined by confocal microscopy (TCS SP2; Leica Microsystems). $\left[\mathrm{Ca}^{2+}\right]_{i}$ levels were analyzed by Leica confocal software (Leica Microsystems) and represented by fluorescent intensity.

Lysosomal stability assessment. Lysosomal stability assessment was performed by using LysoTrack Red (Molecular Probes) that accumulates in lysosomes on the basis of low pH. Cells were incubated with $50 \mathrm{nM}$ LysoTrack Red for $30 \mathrm{~min}$. Nuclei were stained with DAPI and cells were examined by confocal microscopy (TCS SP2; Leica Microsystems).

Targeting construct. Mouse genomic DNA for vector construction was extracted from ES cells that were from the 129/SvcJ7 strain. An approximately $2.5 \mathrm{~kb} \mathrm{Kpnl/Xbal} \mathrm{genomic} \mathrm{fragment} \mathrm{containing} \mathrm{a} \mathrm{part} \mathrm{of} \mathrm{exon} 1$ and an approximately $3 \mathrm{~kb}$ Sall/Notl fragment containing a part of exon 2 were subcloned into the pPNT plasmid (provided by Dr. Pandolffi, Sloan-kettering Institute for Cancer Research, New York, NY, USA). Both fragments were cloned on both sides of a neomycin-resistance cassette (Figure $5 \mathrm{a}$ ), and the targeting vecto was linearized with Notl.

Targeting/genotyping. Electroporation of the construct into ES cells from the 129/SvcJ7 strain (passage 13) and selection for homologous recombinants were performed according to standard procedures. We screened targeted ES colonies using Southern blot and generated chimeric mice. These were mated with C57BL/ $6 \mathrm{~J}$ wild-type mice, and the offspring were genotyped using PCR. Primers $A$ (5'-ACTCAGGCAATCCTCAAGTG-3') and B (5'-AGAGAAATGGAAGAAGCCGC ACTA- $3^{\prime}$ ) were used to detect wild-type alleles. The targeted allele was detected using primers $A$ and $C$ ( $5^{\prime}$-GCGTGCAATCCATCTTGTTC- $\left.3^{\prime}\right)$.

Histological analysis of placentae. Placentae isolated from pregnant mice were fixed in $4 \%$ paraformaldehyde, embedded in paraffin, and serially sectioned at $5 \mu \mathrm{m}$. The sections were stained with hematoxylin and eosin as per standard procedures. Sections for the detection of endogenous alkaline phosphatase activity were prepared as described previously, ${ }^{32}$ and incubated with 5-bromo-4-chloro-3-indolylphosphate/nitroblue tetrazolium in Tris buffer $\mathrm{pH}$ 9.5). Alkaline phosphatase activity resulted in blue staining. For PCNA immunohistochemical staining, sections were de-paraffinized using xylene, and rehydrated in a graded series of ethanol with a final wash in distilled water. Antigen retrieval was carried out by boiling the sections in $10 \mathrm{mM}$ citrate buffer ( $\mathrm{pH}$ 6.0) for $15 \mathrm{~min}$. Endogenous peroxidase activity was blocked by treating the sections with $3 \% \mathrm{H}_{2} \mathrm{O}_{2}$, followed by incubation with mouse monoclonal anti-PCNA antibody (Santa Cruz Biotechnology) at $4^{\circ} \mathrm{C}$ overnight. After being washed with PBS, the primary antibody was detected using the ChemMate DAKO EnVision Detection kit, peroxidase/DAB, rabbit/mouse (DAKO, Glostrup, Denmark) according to the manufacturer's protocol. The sections were then counterstained with hematoxylin, dehydrated, and mounted.

Statistical analysis. The statistical significance of difference between various groups was analyzed by using analysis of variance (ANOVA) and Student-NewmanKeuls test for multiple comparisons. Statistical analysis was performed using the statistical software SPSS11.5. Differences among groups were regarded as significant if $P<0.05$.
Acknowledgements. We thank Dr. Pandolffi for generously providing the targeting vector. This work was supported by grants from the Key Programs of the National Natural Science Foundation of China (No. 30330350) and the Shanghai Committee of Science and Technology (No. 04XD14015).

1. Zhao X, Li J, He Y, Lan F, Guo J, Zhao R et al. A novel growth suppressor gene on chromosome 17p13.3 with a high frequency of mutation in human hepatocellular carcinoma. Cancer Res 2001; 61: 7383-7387.

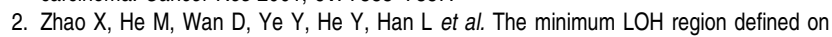
chromosome 17p13.3 in human hepatocellular carcinoma with gene content analysis. Cancer Lett 2003; 190: 221-232.

3. Xiao W, Park CK, Park JY, Lee JH, Kim HS, Cho YG et al. Genetic alterations of the HCCS1 gene in Korean hepatocellular carcinoma. APMIS 2003; 111: 465-673.

4. Yang J, Liu X, Bhalla K, Kim CN, Ibrado AM, Cai J et al. Prevention of apoptosis by Bcl-2: release of cytochrome $c$ from mitochondria blocked. Science 1997; 275: 1129-1132.

5. Gross A, Jockel J, Wei MC, Korsmeyer SJ. Enforced dimerization of BAX results in its translocation, mitochondrial dysfunction and apoptosis. EMBO J 1998; 17: 3878-3885.

6. Jürgensmeier JM, Xie Z, Deveraux Q, Ellerby L, Bredesen D, Reed JC. Bax directly induces release of cytochrome $c$ from isolated mitochondria. Proc Natl Acad Sci USA 1998; 95: 4997-5002

7. Cao X, Deng X, May WS. Cleavage of Bax to p18 Bax accelerates stress-induced apoptosis, and a cathepsin-like protease may rapidly degrade p18 Bax. Blood 2003; 102: 2605-2614.

8. Sawada M, Hayes $\mathrm{P}$, Matsuyama $\mathrm{S}$. Cytoprotective membrane-premeable peptides designed from the Bax-binding domain of Ku70. Nat Cell Biol 2003; 5: 352-357.

9. Chwieralski CE, Welte T, Bühling F. Cathepsin-regulated apoptosis. Apoptosis 2006; 11: 143-149.

10. Bidère $\mathrm{N}$, Lorenzo HK, Carmona S, Laforge M, Harper F, Dumont $\mathrm{C}$ et al. Cathepsin D triggers Bax activation, resulting in selective apoptosis-inducing factor (AIF) relocation in T lymphocytes entering the early commitment phase to apoptosis. J Biol Chem 2003; 278 : 31401-31411.

11. Nomura M, Shimizu S, Sugiyama T, Narita M, Ito $T$, Matsuda $H$ et al. 14-3-3 Interacts directly with and negatively regulates pro-apoptotic Bax. J Biol Chem 2003; 278 : 2058-2065.

12. Sawada M, Sun W, Hayes $P$, Leskov K, Boothman DA, Matsuyama S. Ku70 suppresses the apoptotic translocation of Bax to mitochondria. Nat Cell Biol 2003; 5: 320-329.

13. Susin SA, Lorenzo HK, Zamzami N, Marzo I, Snow BE, Brothers GM et al. Molecular characterization of mitochondrial apoptosis-inducing factor. Nature 1999; 397: 441-446.

14. Wang M, Zhang L, Han X, Yang J, Qian J, Hong S et al. Atiprimod inhibits the growth of mantle cell lymphoma in vitro and in vivo and induces apoptosis via activating the mitochondrial pathways. Blood 2007; 109: 5455-5462.

15. Mignotte B, Vayssière J-L. Mitochondria and apoptosis. Eur J Biochem 1998; 252: 1-15.

16. Yoshino T, Kishi H, Nagata T, Tsukada K, Saito S, Muraguchi A. Differential involvement of p38 MAP kinase pathway and Bax translocation in the mitochondria-mediated cell death in TCR- and dexamethasone-stimulated thymocytes. Eur J Immunol 2001; 31: 2702-2708.

17. Ly JD, Grubb DR, Lawen A. The mitochondrial membrane potential $\left(\Delta \psi_{\mathrm{m}}\right)$ in apoptosis; an update. Apoptosis 2003; 8: 115-128

18. Lim ML, Minamikawa T, Nagley P. The protonophore CCCP induces mitochondrial permeability transition without cytochrome $c$ release in human osteosarcoma cells. FEBS Lett 2001; 503: 69-74.

19. Degenhardt K, Sundararajan R, Lindsten T, Thompson C, White E. Bax and Bak independently promote cytochrome $c$ release from mitochondria. J Biol Chem 2002; 277 : 14127-14134.

20. Stridh H, Gigliotti D, Orrenius S, Cotgreave I. The role of calcium in pre- and postmitochondrial events in tributyltin-induced T-cell apoptosis. Biochem Biophys Res Commun 1999; 266: 460-465.

21. Sharma AK, Rohrer B. Calcium-induced calpain mediates apoptosis via caspase-3 in a mouse photoreceptor cell line. J Biol Chem 2004; 279: 35564-35572.

22. Smaili SS, Hsu YT, Sanders KM, Russell JT, Youle RJ. Bax translocation to mitochondria subsequent to a rapid loss of mitochondrial membrane potential. Cell Death Differ 2001; 8: 909-920.

23. Gao G, Dou QP. N-terminal cleavage of bax by calpain generates a potent proapoptotic $18-\mathrm{kDa}$ fragment that promotes bcl-2-independent cytochrome $c$ release and apoptotic cell death. J Cell Biochem 2000; 80: 53-72.

24. Watson ED, Cross JC. Development of structures and transport functions in mouse placenta. Physiology 2005; 20: 180-193.

25. Wu L, de Bruin A, Saavedra HI, Starovic M, Trimboli A, Yang Y et al. Extra-embryonic function of $\mathrm{Rb}$ is essential for embryonic development and viability. Nature 2003; 421: 942-947.

26. Leiwen $\mathrm{H}$, Meinhold-Heerlein I, Oliveira V, Schwarzenbacher R, Luo G, Wadle A et al. Characterization of the human GARP (Golgi associated retrograde protein) complex. Exp Cell Res 2005; 306: 24-34

27. Conibear E, Stevens TH. Vps52p, Vps53p, and Vps54p form a novel multisubunit complex required for protein sorting at the yeast late Golgi. Mol Biol Cell 2000; 11: 305-323. 
28. Pohlmann R, Boeker MW, von Figura K. The two mannose 6 phosphate receptors transport distinct complements of lysosomal proteins. J Biol Chem 1995; 270: 27311-27318.

29. Maianski NA, Roos D, Kuijpers TW. Bid truncation, bid/bax targeting to the mitochondria and caspase activation associated with neutrophil apoptosis are inhibited by granulocyte colony-stimulating factor. J Immunol 2004; 172: 7024-7030.

30. Dörrie J, Gerauer H, Wachter Y, Zunino SJ. Resveratrol induces extensive apoptosis by depolarizing mitochondrial membranes and activating caspase- 9 in acute lymphoblastic leukemia cells. Cancer Res 2001; 61 4731-4739.

31. Ye JL, Mao WP, Wu AL, Zhang NN, Zhang C, Yu YJ et al. Cadmium-induced apoptosis in human normal liver $\mathrm{L}-02$ cells by acting on mitochondria and regulating $\mathrm{Ca}^{2+}$ signals. Environ Toxicol Pharmacol 2007; 24: 45-54.

32. Dempo K, Kottel RH, Fishman WH. Demonstration of species difference of placental alkaline phosphatase isozymes in acetone-fixed paraffin-embedded tissues. $J$ Histochem Cytochem 1980; 28: 282-284.

Supplementary Information accompanies the paper on Cell Death and Differentiation website (http://www.nature.com/cdd) 